\title{
Using R to Collect, Analyze and Visualize Graduate Attribute Data
}

\author{
Jake Kaupp \\ Queen's University \\ jake.kaupp@queensu.ca
}

\begin{abstract}
This paper will cover the use of the opensource software R, a prevalent IDE (Rstudio) and many of the community built packages that can be used in concert to help collect, analyze and visualize graduate attribute data. Using these tools, developing user-focused workflows and applying effective information visualization principles this paper will illustrate how these tools can be used to quickly and effectively share a vast and complex amount of information to faculty and programs. This approach has been effective in building engagement and buy-in by placing the data in the hands of instructors and program committees. This paper will cover approaches to interfacing and leveraging other systems to streamline and unify existing processes.
\end{abstract}

Keywords: Accreditation, Software, Data Processing, Information Visualization

\section{INTRODUCTION}

There is an enormous amount of student data that higher education institutions routinely collect. This data is collected in registration systems, student information systems, learning management systems, curriculum workbooks, calendars, syllabi, surveys as well as data from external sources such as the office of institutional research and planning. This becomes much more pronounced with professional programs such as engineering. The new accreditation criteria have introduced additional complexities as well as volume of data.

As most engineering programs are familiar with, they are required to develop outcomes that reflect student performance in the 12 graduate attributes, map these attributes to courses and programs, assess student performance on these indicators, and the use the results for data-informed program improvement. This seems like a simple enough exercise, but underneath the surface is a very complex and data intensive process. Since a comprehensive off-the-shelf solution isn't readily available, many institutions have been developing their own systems to collect, manage and distribute this data.
Many of these systems represent ad-hoc processes, built to directly address a pressing need and with little attention to the long term sustainability of the process. Programs are making due with what they have, leveraging excel, database systems and manual analysis to meet accreditation requirements. However, these systems are largely inefficient, unsustainable and prone to duplication. Programs are aware of this, and are now looking to develop more efficient and effective processes for collecting, storing, and communicating the results of graduate attribute data.

At Queen's University, a flexible approach to accreditation is employed. The overall process is managed at the Faculty level setting universal standards and guidelines for the programs to follow. Programs are free to develop their own indicators, mapping, and are encouraged to tailor their approach to fit their needs and climate. Data is collected by the programs, in turn processed by the faculty and distributed back to programs and instructors for future improvement.

The collection, analysis, reporting and distribution of all programs graduate attribute data is facilitated by both open-source and common commercial software (Excel). At the core of these processes is an open source, extensible, statistical programming software called $\mathrm{R}$.

\section{2. $\mathbf{R}$}

\subsection{What is $R$ ?}

$\mathrm{R}$ is an open-source language and environment for statistical computing and graphical techniques. It is called an environment to distinguish that it is a planned and coherent system rather than a collection of tools.

It is a GNU project, purely open source and is highly extensible. This is one of the core strengths of $R$, in which users can extend the capabilities of the system through the development of packages which broadly increase and enhance the value of the system [1].

Originally, R was the domain of of statistics and was used for analyses and high quality graphics. Recently it has become the flagship for reproducible research, data science, information visualization and reporting. 
$\mathrm{R}$ is a scripting language, permitting the automation of typical task based work. It is most often used for the reading, wrangling, analysis, modelling and communication of data. It was this core functionality that lead to Queen's using R, as the graduate attribute process (represented simplistically) is reflective of what the $\mathrm{R}$ language is best known for.

\subsection{RStudio}

$\mathrm{R}$ is a simple command line interface, and scripts can be created via text-based editors such a vim or emacs. While this approach is suitable, there are other tools that supplement and empower users of $\mathrm{R}$ by providing an integrated development environment (IDE). One of the most popular IDE for R is RStudio and open-source IDE provided by RStudio Inc [2] (www.rstudio.com).

RStudio provides a number of visual tools to assist programming in R (environment viewer, plotting devices, file management, version control, history, etc). The majority of this functionality is provided by packages written by the RStudio team. (www.rstudio.com/products/rpackages/)

\subsection{Packages}

Packages are what make R. These are small, self contained operations, functions and compiled code in a highly defined format. In R, packages comprise a library that is used to invoke different functionality for base R. The base installation of $\mathrm{R}$ comes with a standard set of packages. User developed packages are available on the Comprehensive R Archive Network (CRAN), and must follow a set of standards and policies to be accepted. Other user developed packages can also be found on github (www.github.com), a web-based collaborative service for programming and development.

\subsection{System-view}

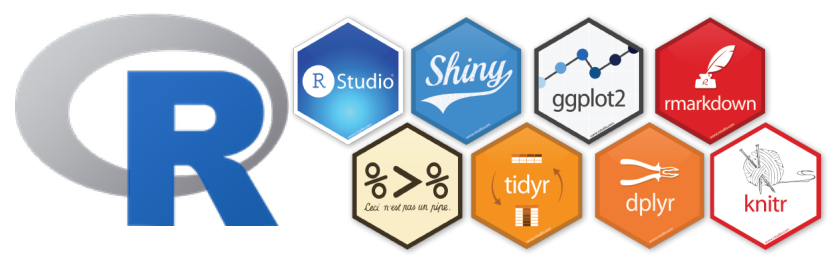

Figure 1: A System-view of R, Rstudio and popular packages

When collectively viewed, all of the pieces provide a set of very powerful tools that can accomplish nearly every needed data management and reporting aspect of the $\mathrm{CEAB}$ accreditation requirements (Figure 1). What began as a approach dedicated to the analysis and visualization of assessment data has soon blossomed into a more integrated approach with $\mathrm{R}$ (and associated pieces) providing the means to leverage multiple sources of student data across campus, provide stock and customized opportunities for analysis, visualize the wealth of information to provide clarity and spur insight, and help alleviate workloads by automating many of the bureaucratic elements of accreditation (e.g. populating CIS sheets, and other accreditation documents).

New developments in R, from RStudio, has taken steps to integrate $\mathrm{R}$ and the web using HTLM5 and popular JavaScript frameworks. The result is a reactive webapplication framework called shiny that permits users to develop applications that utilize $\mathrm{R}$ in but in an interactive and exploratory manner.

\section{WORKFLOWS}

Great tools are useful. Great tools are even more useful when they are intentionally used to create workflows to streamline and assist people in their work. The graduate attribute process, at its core, is a data science problem. Data science is a buzzworthy term for a thirty-year-old field, what data science is defined as:

"an interdisciplinary field about processes and systems to extract knowledge or insights from data in various forms" [3], [4]

What a data science problem looks like is illustrated below in Figure 2.

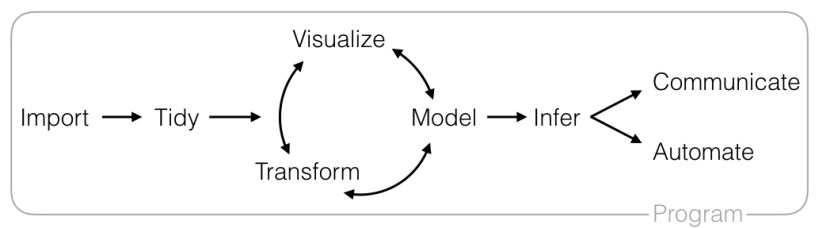

Figure 2:Data Science Workflow github.com/rstudio/RStartHere

The data science workflow frames the problem by importing data from various sources, tidying and cleaning the data for use, then iteratively visualizing, modeling and transforming the data until inferences can be made, communicating the inferences to an audience, and automating it to run on its own.

It shares much in common with a continuous improvement processes cycle, such as the EGAD Continuous Improvement Process illustrated in Figure 3. It is present in nearly every aspect, but is represented more within the latter stages of the cycle. If we move down to a more granular level, you can start to see the different tasks that take place at each stage in a continuous improvement process. Many of these tasks involve instructors and committees doing a lot of manual work: creating outcomes, linking them to assessments, mapping courses, assessing students, collecting data, analyzing it, suggesting improvements. There's also a bit of a meta-level of process 
management, keeping track of what's been decided on and making sure it is chronicled.

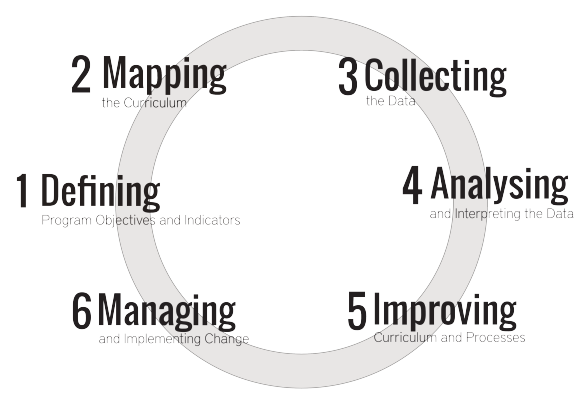

Figure 3: EGAD Continuous Improvement Process

If you want to design a system that people are going to use, you'll want to keep in mind that it has to: 1) do something for them 2) work alongside their usual process. You want to build your tools and systems that minimize the tasks that your users have to accomplish and strive to maximize the benefit for them. You'll want to develop a descriptive workflow for your users to show them what the system will do for them, and the unnecessary steps that it will remove. A sample workflow is outline in Figure 4.

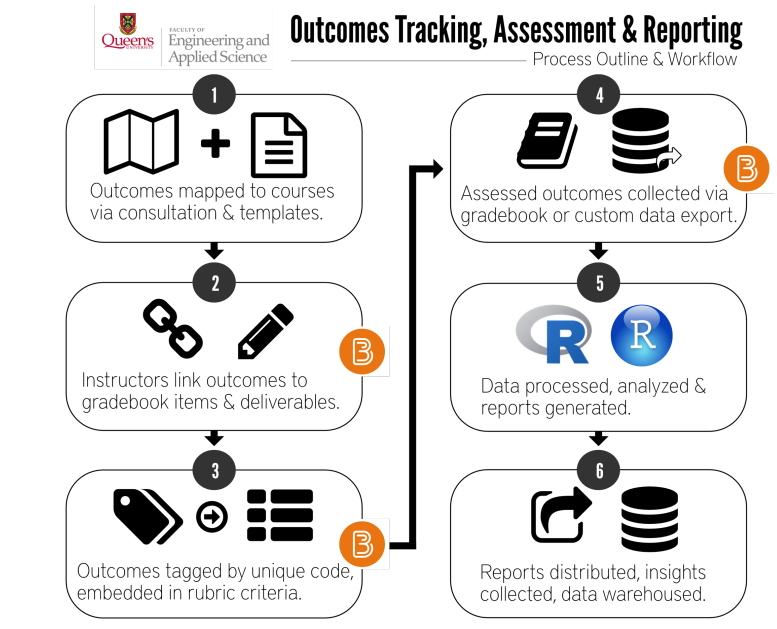

Figure 4: Sample Queen's Workflow

For instance, data collection can be a pain in a templatebased environment. The same with reporting curriculum mapping. The Queen's approach and workflow encourages users to adopt a new learning management system (LMS) and set up their indicators and assessments with educational developers. There is also a common syllabus or course outline they are encouraged to use. If the instructor uses both the tools, with the developer verifying correct usage, it removes the data collection workload for the instructor. The data collection is handled on the back end by a custom data export from the LMS. Curriculum mapping information is extracted from the Syllabi and LMS and can be used downstream.
Additionally, this information is used later on to populate course information sheets for CEAB documentation.

This is a simple tradeoff for the instructor. Invest a little time up front for a reduced workload later on in a busy semester. Instead of them doing the work, programming workflows (as mentioned before) in combination with scheduling tools (GNU mcron), scripting tools (GNU make) and $\mathrm{R}$ functions (packages) are used in conjunction with oversight from an analyst.

\section{QUEEN'S APPROACH}

While the workflows provide a means to describe the expectations for the system as well as provide a means to encourage use of the system, the standalone pieces that comprise the system are still unclear. A better picture of the individual elements ( $\mathrm{R}$ packages and other tools) are illustrated below in Figure 5.

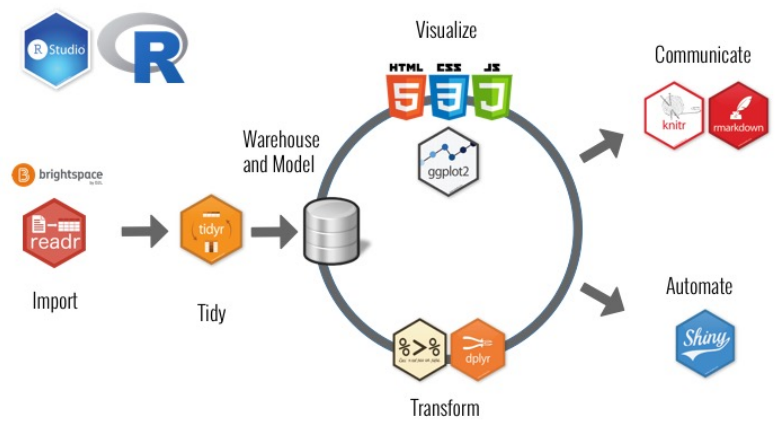

Figure 5: Workflow R Elements used in the Queen's Approach

A more sub-system or architecture focused view of the approach is presented below in Figure 6.

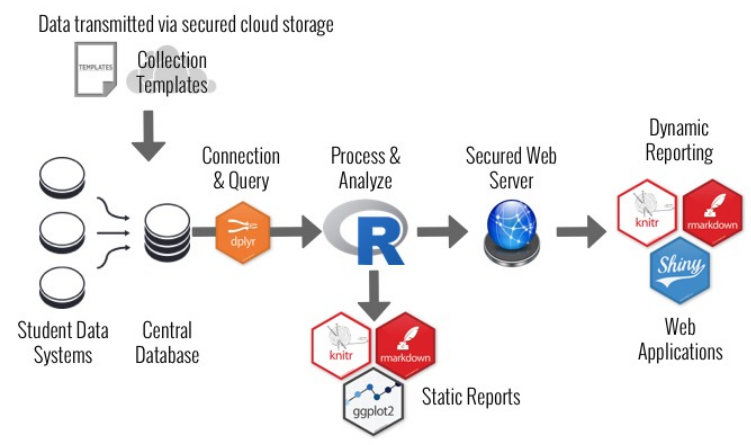

Figure 6: Detail view of the Queen's Approach

The system, QUiVER, is a collection of open source tools and frameworks purposefully designed to be scalable (contingent upon the maturity of other student data systems). It bridges other systems, prioritizing available connections (OBDC, Restful API) over direct integration. When a connection is impractical or impossible, data can be archived and stored for direct use and reporting of 
otherwise inaccessible data (OIRP, Peoplesoft). Data can also be collected in an offline fashion, and processed from a number of data collection templates (any spreadsheet format, word document), which can be customized to fit program needs yet permit proper processing of the data. Processing of this is handled by many data reading packages (readr, readxl, xml, rio).

The core of the system is a scalable open-source database, such as MySQL, MSSQL or MongoDB. This permits the long-term storage and structured environment for the rapid and on-demand analysis, and the environment to dynamically visualized information for exploratory data analysis.

The connection between $\mathrm{R}$ and the database is permitted by a number of database utility packages (RODBC, RJDBC, DBI) and uses the data manipulation package dplyr as a front end on top of the database. The reason for this is that dplyr provides the conversion from easier-toread $\mathrm{R}$ code to an implanted SQL query. The package also provides most SQL functionality in native $\mathrm{R}$ allowing for the server-side processing of the data prior to further analysis. This sidesteps the 'big data' concern by subsetting to medium to small data prior to downstream work.

The majority of the processing and manipulation of the data is performed prior to the data being uploaded to the database. However, there is always the need to at the moment manipulation of the data, which is provided by the tidyr, dplyr and magrittr packages. These 3 packages offer a host of data manipulation routines, from transforming data from wide to long formats, database style joins, variable creation, grouped operations and summaries. There is also a catch-all functional programming package, purrr, to facilitates a number of repeated procedures and steps on large collections of data (e.g. keying a data table on a course, and repeating the same function for each one), that is essential for pre-processing reports and modelling data.

Once the data is processed and analyzed, it is then visualized and reported. The bulk of this work is provided by the knitr and rmarkdown packages which provide the ability to create literate programming reports that blend text and code. The rmarkdown package is the the markdown language specifically tailored for $\mathrm{R}$, allowing the easy creation of HTML documents straight from text. The knitr package harnesses the templating power of pandoc to render markdown into docx or pdf formats using LaTex. It also provides the interpreter for the native $\mathrm{R}$ code, permitting the inclusion of the high quality graphics the platform is known for.

The visualization of the data is handled largely by ggplot2[5]. The 'gg' in the package name stands for the "grammar of graphics", an abstraction created by famed statistician Leland Wilkinson[6]. It seeks to make effective plotting easily by following a layered grammar of graphics. Each plot is created by data and aesthetic mappings, geometric objects, scales, guides, coordinates and statistical transformations. Each of these combine together to form different plot layers, and it is the proper organization and forming of these layers that give rise to lush graphics. This package makes it easier to create meaningful graphics and visualizations as a great deal of effective practise and methods are represented in ggplot2. Programs should be aware that the development of effective visualizations and graphs is a nuanced process. Carelessly designed, redundant or spurious visualizations can cause confusion and impeded insight rather than facilitate it.

Information and meaning-making processes sometimes require a bit of exploration in order to clarify or understand. Having this ability to explore the data in an on-demand and interactive fashion is another intended capability of the system. The shiny and htmlwidgets packages provides an interactive framework for building web applications and brings the power of many JavaScript visualization frameworks (d3js, highcharts, dimple, plotly) to R. Using a secured web-server, users can log into the system and explore the data that they are permitted access to (keyed on login information, uses views in the database as well as shiny access) and allows the to explore the data real-time, at any time. This extended to student performance, curriculum mapping, program level information and historical records.

\section{INFORMATION VISUALIZATION}

Building interest and engagement in the system through making life easier for your users is not the only approach. The sheer amount of data that an instructor produces in assessment activities can quickly overwhelm them. Few instructors ever move beyond plotting the distribution of their grades to really question what is going on. The granularity of the data collected by graduate attribute assessment provides a remarkable opportunity to really explore student performance.

Providing the results of assessment data in an efficient and effective manner to provoke questions and discussion should be the underlying goal of any visualization. The writings of Edward Tufte on visualization hold especially true[7]:

Visualizations should

- $\quad$ consists of complex ideas communicated with clarity, precision, and efficiency.

- is that which gives to the viewer the greatest number of ideas in the shortest time with the least ink in the smallest space.

- $\quad$ show the data

- induce the viewer to think about the substance rather than about methodology, graphic design, the technology.

- avoid distorting what the data have to say 
- $\quad$ present many numbers in a small space

- make large data sets coherent

- $\quad$ encourage the eye to compare different pieces of data

- $\quad$ reveal the data at several levels of detail, from a broad overview to the fine structure

- $\quad$ serve a reasonably clear purpose: description, exploration, tabulation, or decoration

- be closely integrated with the statistical and verbal descriptions of a data set.

Tufte's work provides an excellent set of guidelines to consider when beginning to design graphics. There is also a set of principles or a framework, drawn from research into visual and graphical perception[8]. This work offers insight on how effectively humans can decode the information in graphics using various methods. The results, ranked by method of encoding are shown below in Figure 7.
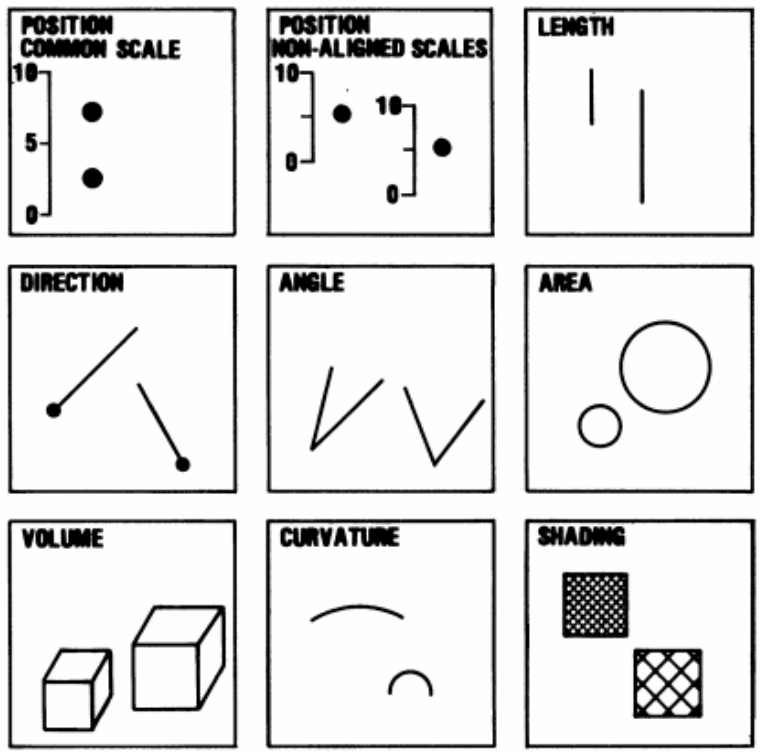

colon satration

Figure 7: Cleveland's Ranking of Decoding Information

There is far more to effective information visualization that just these principles, and in far more depth than what was so briefly covered here. The visualizations used in the Queen's approach integrate these principles in their design and were constructed with a clearly defined objective or key guiding question. They were also iteratively developed, collecting feedback from stakeholders about clarity, utility and meaning. It is strongly urged that anyone developing visualizations learn more about the field before designing their own.

\section{EXAMPLES}

There are many approaches utilized within the Queen's approach, with many of them still in development. The most prominent image is the attribute 'bubble dashboard' (Figure 8), which is meant to summarize cohort performance in indicators for each attribute. It is the 'at-aglance', high level view to provide a sense of where to focus analysis.

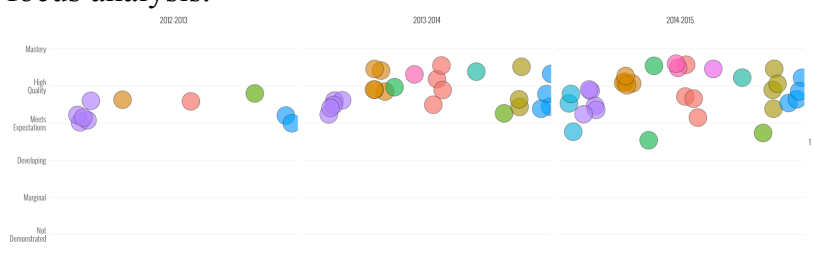

Figure 8: Graduate Attribute Bubble Dashboard

Curriculum mapping is an area sorely in need of visualization. Too long have matrices and tables had the run of the roost. There are multiple alternatives to presenting mapping data, which is essentially a hierarchy and can be visualized by many network principles. Illustrated below is a treemap, which visualizes the frequency of each category and subcategory by areas of the rectangles. In this example, attributes for a single year of a program are mapped. The areas of the rectangles are proportional to the frequency of assessment of an attribute. This can easily illustrate the focus and gaps in a programs development.

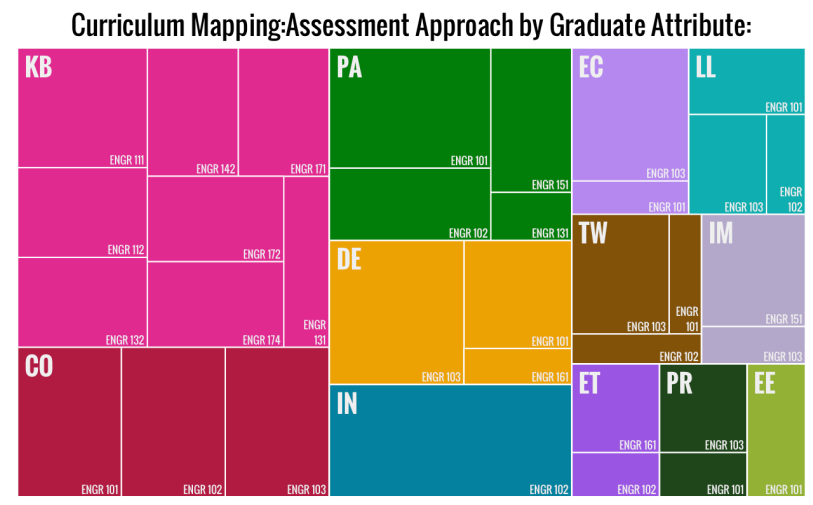

Figure 9: AU and Attribute Curriculum Treemaps

There is also an alternative view for curriculum mapping, which is an interpretation of mapping material provided by CEAB, shown below in Figure 10. This is a rework of a stacked-bar depiction of an IDA map that focused on how many courses introduce, develop or advance an attribute. 


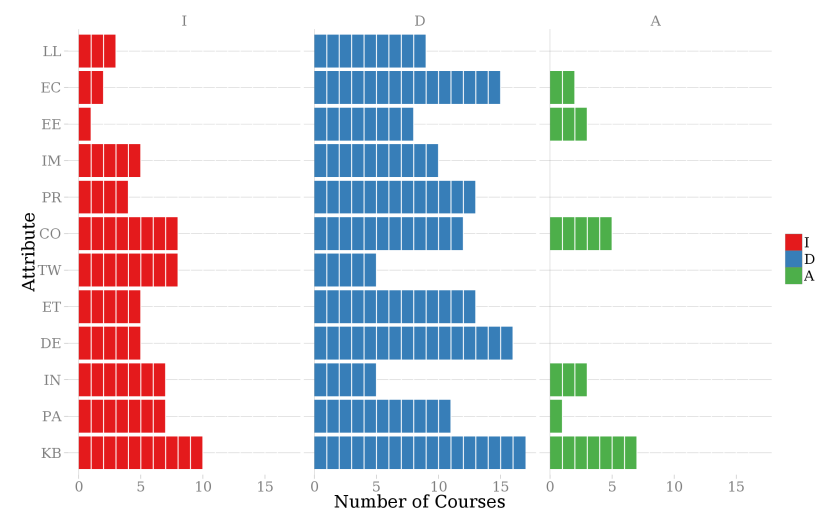

Figure 10: Reworking of the CEAB IDA Map Visualization

As student performance is a key area for many of the visualizations, the types of visualizations presented can range from the simple histogram shown in Figure 11, to the slopegraph presenting student growth in communications outcomes in Figure 12, to the results contrasting a rubric used over two years of the program to assess critical thinking in engineering students in Figure 13. These images contain a great deal of information, each presented at different levels of aggregation and granularity to illustrate the different questions that each depiction can prompt.

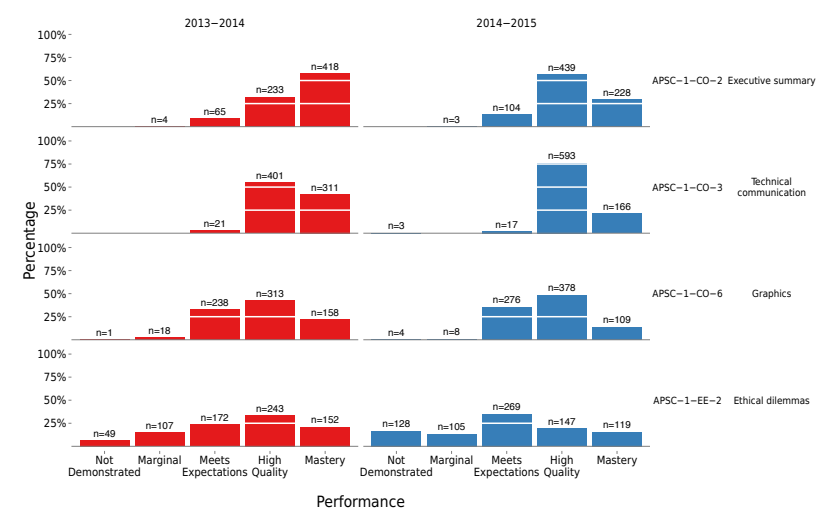

Figure 11: Simple Histograms of Student Performance

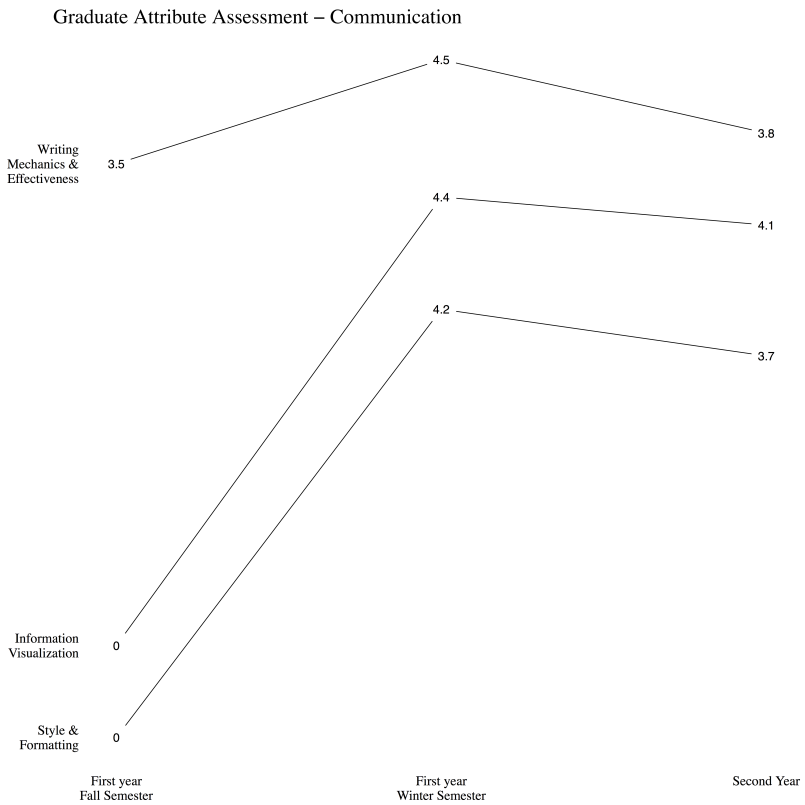

Figure 12: Slopegraph of growth in communication attribute

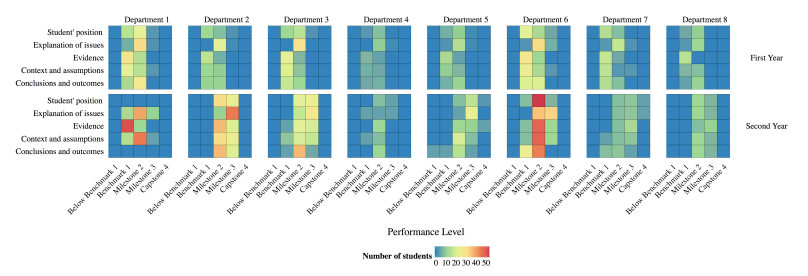

Figure 13: Heatmap of Critical Thinking Performance

This final image was included as an illustration of the types of multifaceted, complex visualization that can be produced within $\mathrm{R}$ and its packages (Figure 14). This is a depiction of results from a project that was evaluating student abilities in critical thinking, problem solving and written communication using a standardized tests and specialized rubrics. This presents the results for a cohort in an engineering program. The darker lines illustrate the mean scores (and associated growth), and the lighter grey lines are the growth of the individual students, providing a sense of variance underlying the data.

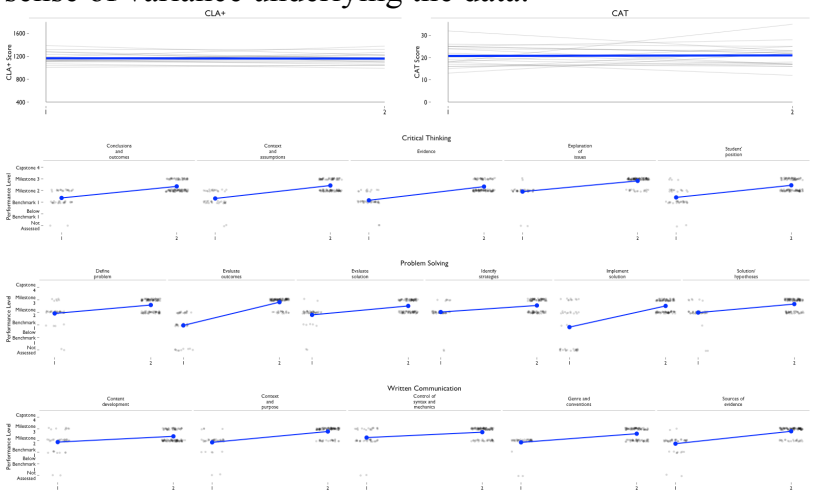

Figure 14: Complex Infographic Style 


\section{CONCLUSIONS}

This paper presented the use of R, Rstudio and community developed packages together with other opensource elements to provide a system for the collection, analysis, reporting and visualization of graduate attributes. It also provides some areas to focus on for those seeking to develop their own system: flexible workflows, identifying and communicating with data stores and designing graphics using effective principles of information visualization.

The community for $\mathrm{R}$ is very large, incredibly welcoming and very helpful. It has an active message board, many google groups and is growing more and more on stackoverflow. It is one of the fastest growing languages for data science and modern data problems. It is a very powerful and flexible language, with a great deal of promise. It is not without its downfalls, yet many of the packages use a external code base (fortran, $\mathrm{C}++$ ) to shore up potential performance and speed issues by writing compiled code directly in an interpreted language.

Preliminary and rudimentary code for many of these graphics can be found at http://github.com/jkaupp.

\section{REFERENCES}

[1] R. C. Team, The R project for statistical computing. Vienna [The R Project for Statistical Computing, 2014.

[2] R. S. Team, RStudio: integrated development for $R$. RStudio, 2014.

[3] W. S. Cleveland, "Data Science: An Action Plan for Expanding the Technical Areas of the Field of Statistics," International Statistical Review/Revue Internationale de Statistique, vol. 69, no. 1, pp. 21-26, Apr. 2001.

[4] V. Dhar, "Data science and prediction," Communications of the ACM, vol. 56, no. 12, pp. 64-73, Dec. 2013.

[5] H. Wickham, "A Layered Grammar of Graphics," http://dx.doi.org.proxy.queensu.ca/10.1198/jcgs.2 009.07098, vol. 19, no. 1, pp. 3-28, Jan. 2012.

[6] L. Wilkinson, The Grammar of Graphics. Springer Science \& Business Media, 2013.

[7] E. R. Tufte, The Visual Display of Quantitative Information. 2001.

[8] W. S. Cleveland and R. McGill, "Graphical perception: Theory, experimentation, and application to the development of graphical methods," Journal of the American statistical ..., 1984. 